\title{
Photoionisation of Atoms and Möller Operators
}

\author{
Philippe Combe \\ Université d'Aix-Marseille II, UER Scientifique de Luminy et Centre de Physique Théorique, \\ CNRS, Marseille, France
}

\author{
Eric Mourre and Jean-Louis Richard \\ Centre de Physique Théorique, CNRS, Marseille, France
}

Received January 22, 1975

\begin{abstract}
The motion of an hydrogenoid atom in a laser field is usually given by the time-dependent hamiltonian $\mathrm{H}(t)=[\boldsymbol{p}-\boldsymbol{A}(t)]^{2} / 2+\mathrm{V}(r)$ where $\mathrm{V}(r)$ is the atomic potential while $\boldsymbol{A}(t)$ is to be connected with the laser field. The existence and unicity for the Cauchy problem of the solutions of the corresponding Schrödinger equation are established under mild conditions on $\boldsymbol{A}(t)$ and $\mathrm{V}(r)$. The existence of Möller operators is investigated in two cases, namely, when the laser field is a function of time only and when it vanishes asymptotically in time. Special attention is paid for the Coulomb case for which a "distorted" Möller operator is derived. Finally, when the laser field vanishes as $t \rightarrow \infty$, the photoionisation probability is properly defined by means of the Möller operator
\end{abstract}

$$
\Omega\left(\mathrm{H}_{\mathrm{A} t}, \mathrm{H}\right)=\underset{t \rightarrow \infty}{\mathrm{s}-\lim _{t \rightarrow}} U_{\mathrm{A} t}(t)^{-1} U(t),
$$

where $U(t)$ is the evolution operator for the system while $U_{\mathrm{At}}(t)$ is the evolution operator for the atom.

\section{Introduction}

The multiphotoionisation of hydrogenoïd or rare gaz atoms by a laser beam has received special attention both experimentally [1] and theoretically [2]. The availability of higher and higher intense beams leads us to abandon the perturbative expansion approach. Other kinds of approximation have been proposed [3] in the framework of a semi-classical treatment in which the photon beam is regarded as a classical external field. However, the non perturbative approximations are motivated by arguments based on pure classical mechanics. We then feel it necessary to have a global analysis of the Schrödinger equation used to describe the multiphotoionisation process. This is the aim of this note.

It is physically reasonable to assume that the system under consideration can be reduced to the interaction of an electron with a central potential (the atomic potential seen by the electron) and with the external radiation field. More precisely the hamiltonian to be considered will be written as

$$
\mathrm{H}(t)=\frac{1}{2}[\boldsymbol{p}-\boldsymbol{A}(t)]^{2}+\mathrm{V}
$$

when expressed in atomic units, where $\mathrm{V}$ denotes the central potential while $\boldsymbol{A}(t)$ is the electromagnetic potential for the radiation field.

In Section II, one proves the existence of solutions of the Schrödinger equation and their unicity for the Cauchy problem [4], under rather mild conditions on $\mathrm{V}$ and $\boldsymbol{A}(t)$. 
The following two sections are concerned with two different aspects. In Section III, we are dealing with the scattering problem that is, with the existence of the Möller operator

$$
\Omega\left(\mathrm{H}, \mathrm{H}_{\mathrm{E}}\right) \equiv \mathrm{s}_{t \rightarrow \infty} \lim _{\rightarrow} U(t)^{-1} U_{\mathrm{E}}(t),
$$

where $U(t)$ denotes the unitary evolution operator of the system under the hamiltonian $\mathrm{H}(t)$ while $U_{\mathrm{E}}(t)$ is the evolution operator for an electron in the external field.

The Möller operator $\Omega\left(\mathrm{H}, \mathrm{H}_{E}\right)$ is shown to exist in the case of a pure timedependent electric field without any assumption on its behaviour as $t$ tends to infinity. The central potential V is assumed to be square-integrable or of Hack's type. Also special attention has been paid to the Coulomb case and distorted Möller operators have been defined in a way to be connected with Dollard's approach [5] to Coulomb scattering.

In Section IV, the ionisation problem is considered. We define the photoionisation probability when the laser beam vanishes asymptotically in time, by investigating the existence of the Möller operator

$$
\Omega\left(\mathrm{H}, \mathrm{H}_{\mathrm{A} t}\right)=\mathrm{s}-\lim _{t \rightarrow+\infty} U(t)^{-1} \exp \left(-i t \mathrm{H}_{\mathrm{A} t}\right)
$$

and

$$
\Omega\left(\mathrm{H}_{\mathrm{A} t}, \mathrm{H}\right)=\operatorname{s}_{t \rightarrow+\infty} \operatorname{limpit} \mathrm{H}_{\mathrm{A} t} U(t),
$$

where $\mathrm{H}_{\mathrm{A} t}$ is defined to be the atomic system hamiltonian.

\section{The Evolution}

We intend to investigate the evolution of a system governed by the timedependent hamiltonian

$$
\mathrm{H}(t)=\frac{1}{2}[\boldsymbol{p}-\boldsymbol{A}(t)]^{2}+\mathrm{V}
$$

to be defined in $\mathscr{L}^{2}\left(\mathbb{R}^{3}\right)$. We denote by $p_{i}, i=1,2,3$, the differential operator $-i \frac{\partial}{\partial x_{i}}$. We shall assume that

(i) $\mathrm{V} \in \mathscr{L}^{2}\left(\mathbb{R}^{3}\right)+\mathscr{L}^{\infty}\left(\mathbb{R}^{3}\right)$.

(ii) $\mathrm{A}_{i}(t, \boldsymbol{x}), i=1,2,3$ are real functions, differentiable relative to $\boldsymbol{x}$.

(iii) $\mathrm{A}_{i}(t)$ and $\partial i \mathrm{~A}_{i}(t)$ are bounded operators, uniformly in $t$.

(iv) the maps $t \rightarrow \mathrm{A}_{i}(t)$ and $t \rightarrow \partial_{i} \mathrm{~A}_{i}(t)$ are strongly differentiable and $\frac{\partial}{\partial t} \mathrm{~A}_{i}(t)$ and $\frac{\partial}{\partial t} \partial_{i} \mathrm{~A}_{i}(t)$ are bounded.

Under assumptions (i)-(iii), the operator

$$
\mathrm{B}(t)=\frac{1}{2}[\boldsymbol{p} \cdot \boldsymbol{A}(t)+\boldsymbol{A}(t) \cdot \boldsymbol{p}]+\frac{1}{2} \boldsymbol{A}(t)^{2}+\mathrm{V}
$$


is symmetric and Kato-tiny [6] relative to $H_{0}$, the self adjoint extension of $-\Delta$. Then from the Kato-Rellich theorem [7], one can assert that $\mathrm{H}(t)$ is a self-adjoint operator with domain $\mathscr{D}\left(\mathrm{H}_{0}\right)$. We see in particular that the domain of $\mathrm{H}(t)$ is independent of $t$.

Under the assumptions (i)-(iv), we get

Lemma 1. a) The operator $\mathrm{B}(t)$ is Kato-tiny relative to $\mathrm{H}_{0}$ uniformly in $t$, i.e., given a arbitrarily small, there exists $b>0$ such that

$$
\|\mathrm{B}(t) \psi\| \leqq a\left\|\mathrm{H}_{0} \psi\right\|+b\|\psi\| \quad \text { for all } \quad \psi \in \mathscr{D}\left(\mathrm{H}_{0}\right)
$$

on any finite interval of time. And there exists $\mathrm{E}_{0}$ such that $\mathrm{H}(t) \geqq-\mathrm{E}_{0}+1$.

b) $\left[\mathrm{H}(t)+\mathrm{E}_{0}\right]^{-1}$ is strongly differentiable and $\left\|\left[\mathrm{H}(t)+\mathrm{E}_{0}\right] \frac{d}{d t}\left[\mathrm{H}(t)+\mathrm{E}_{0}\right]^{-1}\right\|$ is bounded.

Statement a) is easily derived using assumption (iii). The existence of an $E_{0}$ such that $\mathrm{H}(t)+\mathrm{E}_{0} \geqq 1$ is a direct consequence of the uniform Kato-tiny property of $\mathrm{B}(t)$. Note also that the solvent

$$
\mathrm{R}(t)=\left[\mathrm{H}(t)+\mathrm{E}_{0}\right]^{-1}
$$

exists and has norm less than 1.

Statement b) is a direct consequence of assumptions (iii) and (iv).

Yoshida's theorem [8] applies and one then proves the existence of a unitary evolution operator defined by

$$
\psi(t)=U\left(t, t_{0}\right) \psi, \quad t \geqq t_{0}
$$

which can be extended to all of $\mathscr{L}^{2}\left(\mathbb{R}^{3}\right)$. The family of the strongly continuous unitary operators $U\left(t, t_{0}\right)$ has the following properties

$$
\begin{aligned}
U(t, t) & =\mathbb{1} \\
U(t, s) U\left(s, t_{0}\right) & =U\left(t, t_{0}\right) \text { for } t_{0} \leqq s \leqq t .
\end{aligned}
$$

Moreover $U\left(t, t_{0}\right)$ is strongly differentiable on $\mathscr{D}\left(\mathrm{H}_{0}\right)$ and such that for any $t \geqq t_{0}$ and $\psi \in \mathscr{D}\left(\mathrm{H}\left(t_{0}\right)\right)=\mathscr{D}\left(\mathrm{H}_{0}\right)$

$$
\frac{d}{d t} U\left(t, t_{0}\right) \psi=-i \mathrm{H}(t) U\left(t, t_{0}\right) \psi
$$

\section{Existence of the Möller Operator in the Case of a Uniform Electric Field}

We now restrict ourselves to the case of an electric field depending only on time. The vector potential in (1) is chosen to be a differentiable real function of $t$ and therefore $\mathrm{A}_{i}(t)$ is the multiplication by a real number for every $t$.

Let us recall that the "free" motion of the particle is governed by the hamiltonian

$$
\mathrm{H}_{\mathrm{E}}(t)=\frac{1}{2}[\boldsymbol{p}-\boldsymbol{A}(t)]^{2} .
$$


And one can see that the corresponding unitary propagator is then

$$
U_{\mathrm{E}}(t)=\exp -i\left\{\frac{p^{2}}{2} t-\alpha(t) \cdot \boldsymbol{p}+\beta(t)\right\},
$$

where

$$
\begin{aligned}
& \alpha_{i}(t)=\int_{0}^{t} \mathrm{~A}_{i}(\mathrm{~s}) d s \\
& \beta(t)=\frac{1}{2} \int_{0}^{t} A(s)^{2} d s
\end{aligned}
$$

with initial conditions taken at $t=0$.

We want to investigate the existence of the Möller operator defined as

$$
\Omega\left(\mathrm{H}, \mathrm{H}_{\mathrm{E}}\right)=\operatorname{s-lim}_{t \rightarrow+\infty} U(t)^{-1} U_{\mathrm{E}}(t) .
$$

We use a well known lemma [9] essentially based on the existence of the integral

$$
\mathrm{I}(\psi, \tau)=\int_{\tau}^{+\infty}\left\|\frac{\partial}{\partial t} U^{-1}(t) U_{\mathrm{E}}(t) \psi\right\| d t
$$

when $\psi$ belongs to some dense set of $\mathscr{L}^{2}\left(\mathbb{R}^{3}\right)$.

As it has been done when $U_{\mathrm{E}}(t)$ is the actual free motion [9], one can derive an estimate on $\left\|\frac{\partial}{\partial t} U(r)^{-1} U_{\mathrm{E}}(r) \psi\right\|$ for $U_{\mathrm{E}}(t)$ given in (8). This estimate follows from the representation

$$
\left[U_{\mathrm{E}}(t) \psi\right](\boldsymbol{x})=(2 i \pi t)^{-3 / 2} e^{-i \beta(t)} \int d^{3} y e^{i[\boldsymbol{x}+\boldsymbol{\alpha}(t)-\boldsymbol{y}]^{2} / 2 t} \psi(\boldsymbol{y}), \quad t>0
$$

for all $\psi \in \mathscr{L}^{1}\left(\mathbb{R}^{3}\right) \cap \mathscr{L}^{2}\left(\mathbb{R}^{3}\right)$.

Theorem 1. If $\mathrm{V} \in \mathscr{L}^{2}\left(\mathbb{R}^{3}\right)$, then $\Omega\left(\mathrm{H}, \mathrm{H}_{\mathrm{E}}\right)=\mathrm{s}_{t \rightarrow+\infty} U(t)^{-1} U_{\mathrm{E}}(t)$ exists.

Note that one could as well use gaussian functions, the finite sums of which are dense in $\mathscr{L}^{2}\left(\mathbb{R}^{3}\right)$, to get the existence of $\Omega\left(\mathrm{H}, \mathrm{H}_{\mathrm{E}}\right)$ for potentials of Hack's type, i.e., such that $(1+|\boldsymbol{x}|)^{-1 / 2+\varepsilon} \mathrm{V} \in \mathscr{L}^{2}\left(\mathbb{R}^{3}\right)$.

Note also that the proof for the strong limit of $U(t)^{-1} U_{\mathrm{E}}(t)$ as $t$ tends to $-\infty$ can be derived along the lines developed by Prugovecki and Tip [10].

When the central potential V corresponds to Coulomb forces it is well known [5] that one cannot construct the Möller operators in the usual sense. Dollard however has introduced a substitute which in our case has to be modified. We propose to define a unitary "distorted free" propagator $U_{\mathrm{EC}}(t)$ according to

$$
U_{\mathrm{EC}}(t)=U_{\mathrm{E}}(t) \exp -i \mathrm{D}(t),
$$

where the operator $\mathrm{D}(t)$ is assumed to be defined on the set $\mathscr{S}_{\mathrm{C}}$ of functions belonging to $\mathscr{S}\left(\mathbb{R}^{3}\right)$ whose Fourier transform vanishes in some neighbourhood of the axis of the electric field $\boldsymbol{E}$ assuming that $\boldsymbol{E}$ has a fixed direction. The action of $\mathrm{D}(t)$ is given by

$$
[\mathrm{D}(t) \tilde{f}](\boldsymbol{k})=\int_{t_{0}}^{t} d s\left[(\boldsymbol{k} s-\boldsymbol{\alpha}(s))^{2}\right]^{-1 / 2} \tilde{f}(\boldsymbol{k})
$$


where $\tilde{f}$ denotes the Fourier transform of $f \in \mathscr{S}_{\mathrm{C}}$. On one hand, $\mathscr{S}_{\mathrm{C}}$ is dense in $\mathscr{L}^{2}\left(\mathbb{R}^{3}\right)$, on the other hand, $\exp -i \mathrm{D}(t)$ can be defined as the multiplication operator on $\mathscr{L}^{2}\left(\mathbb{R}^{3}\right)$ by a function defined almost everywhere in $\mathbb{R}^{3}$ and of modulus one.

The expression of $\mathrm{D}(t)$ is suggested by an heuristic argument similar to the argument by Buslaev and Matveev [12] for Coulomb like scattering.

In classical mechanics, the Lagrange equations are

$$
\frac{d}{d t} \boldsymbol{p}=\frac{\boldsymbol{x}}{r^{3}} \text { and } \boldsymbol{p}=\dot{\boldsymbol{x}}+\boldsymbol{A}(t) \text {. }
$$

These equations show that for large $r$ the motion of the particle is approximately given by

$$
\boldsymbol{x}=\boldsymbol{p} t-\alpha(t) .
$$

Substituting $\boldsymbol{x}$ by its approximate expression in the Hamiltonian of the particle, one gets

$$
\mathrm{H}(t) \approx \frac{1}{2}[p-A(t)]^{2}+[p t-\alpha(t)]^{-1 / 2}
$$

which will give in quantum mechanics the asymptotic motion of the particle by means of the evolution operator (13).

We want to show that the lemma given in [9] can be applied to the distorted operator

$$
\Omega_{\mathrm{C}}(t)=U(t)^{-1} U_{\mathrm{EC}}(t) .
$$

One sees that

$$
\left\|\frac{\partial}{\partial t} \Omega(t) f\right\|=\left\|\left[V+\frac{\partial}{\partial t} \mathrm{D}(t)\right] U_{\mathrm{EC}}(t) f\right\|, \quad f \in \mathscr{S}_{\mathrm{C}},
$$

where now $\mathrm{V}$ is the Coulomb potential, $\mathrm{V}(\boldsymbol{x})=-1 /|\boldsymbol{x}|$.

Following Dollard, we expect $U_{\mathrm{EC}}(t) f$ to be decomposed into the sum of two functions $f_{1}(t)$ and $f_{2}(t)$ for any $f$ in $\mathscr{S}_{\mathrm{C}}$. Since $\frac{\partial}{\partial r} \mathrm{D}(t) f$ belongs to $\mathscr{S}_{\mathrm{C}}$ for $f$ in $\mathscr{S}_{\mathrm{C}}$, we shall have a similar decomposition which will be written as

$$
U_{\mathrm{EC}}(t) \frac{\partial}{\partial t} \mathrm{D}(t) f=h_{1}(t)+h_{2}(t)
$$

This decomposition is required to fulfill the following two equations

$$
\mathrm{V} f_{1}(t)+h_{1}(t)=0
$$

and

$$
\int_{\tau}^{+\infty}\left\|\mathrm{V} f_{2}(t)+h_{2}(t)\right\| d t<\infty \quad \text { for some } \quad \tau>0 .
$$


This is precisely stated in the following

Lemma 2. a) Let $g \in \mathscr{S}_{\mathrm{C}}$. Then, $U_{\mathrm{EC}}(t) g$ can be decomposed into

$$
U_{\mathrm{EC}}(t) g=g_{1}(t)+g_{2}(t)
$$

with

$$
g_{1}(t)(\boldsymbol{x})=(-i t)^{-3 / 2} e^{-i \beta(t)} \varphi(\boldsymbol{x}, t) \tilde{g}[(\boldsymbol{x}+\alpha(t) / t],
$$

where

$$
\varphi(\boldsymbol{x}, t)=e^{i[\boldsymbol{x}+\boldsymbol{\alpha}(t)]^{2} / 2 t} \exp -i \int_{t_{0}}^{t} d s\left\{\left[(\boldsymbol{x}+\boldsymbol{\alpha}(t) s / t-\boldsymbol{\alpha}(s)]^{2}\right\}^{-1 / 2}\right.
$$

and

$$
g_{2}(t)(x)=(2 i \pi t)^{-3 / 2} e^{-i \beta(t)} e^{i[x+\alpha(t)]^{2} / 2 t} \mathrm{R}_{g}(x+\alpha(t), t),
$$

where

$$
\mathrm{R}_{g}(\boldsymbol{x}, t)=\int d^{3} x^{\prime} e^{-i \boldsymbol{x} \cdot \boldsymbol{x}^{\prime} / t}\left[e^{i \boldsymbol{x}^{\prime 2} / 2 t}-1\right]\left[e^{-i \mathrm{D}(t)} g\right]\left(\boldsymbol{x}^{\prime}\right) .
$$

b) For any $f \in \mathscr{S}_{\mathrm{C}}$ with $h=\frac{\partial}{\partial t} \mathrm{D}(t) f$, Eqs. (18) and (19) hold with the decomposition (21), (23) given above.

The proof of Lemma 2, Part a), is essentially based on the representation (12).

Part b) is derived in a way similar to Dollard's proof given in Ref. [5]. For more details, the reader is referred to [14].

Hence, one can state the

Theorem 1'. Let the "distorted free" evolution operator $U_{\mathrm{EC}}(t)$ be defined as in (13) and let $\mathrm{V}(\boldsymbol{x})$ be the Coulomb potential. Then, the distorted Möller operator

$$
\Omega_{\mathrm{C}}\left(\mathrm{H}, \mathrm{H}_{\mathrm{E}}\right)=\underset{t \rightarrow+\infty}{\mathrm{s}-\lim _{t \rightarrow+\infty}} U(t)^{-1} U_{\mathrm{EC}}(t)
$$

is seen to exist on all of $\mathscr{L}^{2}\left(\mathbb{R}^{3}\right)$.

Finally, let us note that the range of the wave operator $\Omega\left(\mathrm{H}, \mathrm{H}_{\mathrm{E}}\right)$ has an interesting physical meaning in the problem of ionisation. Indeed, let $\psi$ be a vector in the range of this wave operator. Then, there exists a vector $\varphi$ such that $\psi=\Omega\left(\mathrm{H}, \mathrm{H}_{\mathrm{E}}\right) \varphi$ with the property that

$$
\lim _{t \rightarrow+\infty}\left\|U(t) \psi-U_{\mathrm{E}}(t) \varphi\right\|=0 .
$$

That is, for large time the state $U(t) \psi$ behaves as the "free state" $U_{\mathbf{E}}(t) \varphi$ and therefore corresponds to the ionisation of the atom.

\section{The Multiphotoionisation Problem}

In the photoionisation of atoms by a laser beam, the experiment consists in measuring the number of ions which one gets during the time of interaction of the laser beam with the atoms. In this section, we therefore assume that the laser 
beam vanishes as $t$ tends to infinity. Then, asymptotically in time, we are left with the atomic system alone and we have to define the corresponding hamiltonian which we denote by $\mathrm{H}_{\mathrm{A} t}$. In order to have a gauge independent formalism, we formally define $\mathrm{H}_{\mathrm{A} t}$ to be

$$
\mathrm{H}_{\mathrm{A} t}=\frac{1}{2}[\boldsymbol{p}-\boldsymbol{A}(\infty)]^{2}+\mathrm{V},
$$

where $\mathrm{A}_{i}(\infty)$ denotes the multiplication operator

$$
\left[\mathrm{A}_{i}(\infty) \psi\right](\boldsymbol{x})=\mathrm{A}_{i}(\infty, \boldsymbol{x}) \psi(\boldsymbol{x}), \quad \psi \in \mathscr{L}^{2}\left(\mathbb{R}^{3}\right) ; \quad i=1,2,3
$$

with

$$
\mathrm{A}_{i}(\infty, \boldsymbol{x})=\lim _{t \rightarrow+\infty} \mathrm{A}_{i}(t, \boldsymbol{x}) .
$$

In order for this limit to exist, it is enough to assume that v) the electric field $\mathrm{E}_{i}(t, x)$ is such that for $t$ large enough there exists $\mathrm{C}$ and $\mathrm{C}^{\prime}$ for which

(a) $\left\|\mathrm{E}_{i}(t)\right\|=\operatorname{Sup}_{\boldsymbol{x} \in \mathbb{R}^{3}}\left|\mathrm{E}_{i}(t, \boldsymbol{x})\right| \leqq c t^{-\lambda} \quad$ with $\quad \lambda>1 ; \quad i=1,2,3$.

(b) $\left\|\partial_{i} E_{j}(t)\right\|=\operatorname{Sup}_{\boldsymbol{x} \in \mathbb{R}^{3}}\left|\frac{\partial}{\partial x_{i}} \mathrm{E}_{j}(t, \boldsymbol{x})\right| \leqq \mathrm{C}^{\prime} t^{-\lambda^{\prime}} \quad$ with $\quad \lambda^{\prime}>1 ; \quad i=1,2,3$.

With assumption v) (a), $\mathrm{H}_{\mathrm{At}}$ is a well-defined self-adjoint operator with domain $\mathscr{D}\left(\mathrm{H}_{0}\right)$ according to Section I assuming that $\mathrm{A}_{i}(\infty)$ is a bounded operator.

Moreover, using Maxwell equations and assumption v) (b) one can show that there exists a function $\Lambda(x)$ such that

$$
\mathrm{A}_{i}(\infty, x)=\frac{\partial}{\partial x_{i}} \Lambda(x) .
$$

Writing then symbolically the operator $\mathrm{A}_{i}(\infty)$ as $\partial_{i} \Lambda$ one can check that the hamiltonian (27) of the atom is unitarily equivalent to the usual hamiltonian

$$
\mathrm{H}=\frac{1}{2} \boldsymbol{p}^{2}+\mathrm{V}
$$

the unitary transformation being given simply by

$$
\mathrm{W}=\exp i \Lambda \text {. }
$$

Clearly this transformation is to be connected with a gauge transformation.

As a direct consequence, the spectrum of $\mathrm{H}_{\mathrm{A} t}$ is the same as the spectrum of $\mathrm{H}$ so that it is meaningful to ask for the atomic system to be found asymptotically in time in a bound state or in a scattering state.

We are then led to the

Theorem 2. Under assumptions i) ... iv) and if in assumption v) $\lambda>2$, then the Möller operators

$$
\Omega_{t_{0}}\left(\mathrm{H}, \mathrm{H}_{\mathrm{A} t}\right)=s-\lim _{t \rightarrow+\infty} U\left(t, t_{0}\right)^{-1} e^{-i\left(t-t_{0}\right) \mathrm{H}_{\mathrm{A} t}}
$$


and

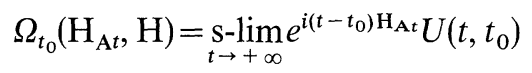

exist on all of $\mathscr{L}^{2}\left(\mathbb{R}^{3}\right)$. Moreover

$$
U\left(t, t_{0}\right)=\Omega_{t}\left(\mathrm{H}, \mathrm{H}_{\mathrm{A} t}\right) e^{-i\left(t-t_{0}\right) \mathrm{H}_{\mathrm{A} t}} \Omega_{t_{0}}\left(\mathrm{H}_{\mathrm{A} t}, \mathrm{H}\right) .
$$

These Möller operators have to be distinguished from the one we defined in Section III. Indeed, no assumption was made about the behaviour in time of the external field. On the contrary, assumption v) indicates that the external field vanishes asymptotically so that we are left with the atomic system alone. It is then meaningful to ask for the existence of the Möller operators $\Omega_{t_{0}}\left(\mathrm{H}, \mathrm{H}_{\mathrm{A} t}\right)$ and $\Omega_{t_{0}}\left(\mathrm{H}_{\mathrm{A} t}, \mathrm{H}\right)$.

We are then in position to define the ionisation probability which is the physical quantity to be measured. Let $\psi$ be the initial state of the system, then after the interaction of the atom with the laser beam has taken place, the state of the system is seen to be $\Omega_{t_{0}}\left(\mathrm{H}_{\mathrm{A} t}, \mathrm{H}\right) \psi$ in the so-called interaction picture. If we denote by $\mathrm{P}_{\mathrm{C}}$ the spectral projection on the absolute continuous subspace relative to $\mathrm{H}_{\mathrm{A} t}$, the ionisation probability will be given by

$$
w_{\psi}\left(t_{0}\right)=\left\|\mathrm{P}_{\mathrm{C}} \Omega_{t_{0}}\left(\mathrm{H}_{\mathrm{A} t}, \mathrm{H}\right) \psi\right\|^{2} .
$$

The first statement of Theorem 2 is quite easily derived, and based on the lemma of Ref. [9]. The existence of the integral

$$
\mathrm{I}_{\mathrm{A} t}(\psi, \tau)=\int_{\tau}^{+\infty}\left\|\frac{d}{d r} U(t)^{-1} e^{-i \mathrm{H}_{\mathrm{A} t} t} \psi\right\| d t
$$

is established once one has noticed that

$$
\left\|\mathrm{p}_{i} e^{-i t \mathrm{H}_{\mathrm{A} t}} \psi\right\| \leqq\left\|\mathrm{p}_{i}\left[\mathrm{H}_{\mathrm{A} t}+i\right]^{-1}\right\|\left\|\left[\mathrm{H}_{\mathrm{A} t}+i\right] \psi\right\|, \quad \psi \in \mathscr{D}\left(\mathrm{H}_{0}\right) .
$$

In Section II, we have shown that the Möller operator $\Omega\left(\mathrm{H}, \mathrm{H}_{\mathrm{E}}\right)$ exists under assumptions i)-iv) when the electric field is a function of time only. Clearly, if we add assumption v) of Section IV, one can show that $\Omega\left(\mathrm{H}, \mathrm{H}_{0}\right)$ exists as well. Indeed, one sees that for the integral

$$
\mathrm{I}_{0}(\psi, \tau)=\int_{\tau}^{+\infty} d t\left\|\mathrm{~B}(t) e^{-i t \mathrm{H}_{0}} \psi\right\|
$$

to exist we can use the estimate

$$
\begin{aligned}
\mathrm{I}_{0}(\psi, \tau) \leqq & \int_{\tau}^{+\infty} d t\left\|\mathrm{~V} e^{-i t \mathrm{H}_{0}} \psi\right\|+\sum_{i=1}^{3} \int_{\tau}^{+\infty} d t\left\|\mathrm{~A}_{i}(t)\right\|\left\|\mathrm{p}_{i} e^{-i \mathrm{H}_{0} t} \psi\right\|+ \\
& +\sum_{i=1}^{3} \int_{\tau}^{+\infty} d t\left\|\mathrm{~A}_{i}(t)\right\|^{2}\|\psi\|, \quad \psi \in \mathscr{D}\left(H_{0}\right)
\end{aligned}
$$

remembering that we had chosen a gauge for which $\mathrm{A}_{i}(t)$ is a function of time only. The first integral is seen to exist for square integrable potentials $\mathrm{V}$ (to be connected with Theorem 1) and for Coulomb like potentials when $\mathrm{H}_{0}$ is replaced by the distorted free hamiltonian (Dollard [5]). The second and third integrals are also finite due to assumption v), the choice of the gauge and the estimate

$$
\left\|\mathrm{p}_{i} e^{-i t \mathrm{H}_{0}} \psi\right\| \leqq\left\|\mathrm{p}_{i}\left[\mathrm{H}_{0}+i\right]^{-1} \psi\right\|\left\|\left[\mathrm{H}_{0}+i\right] \psi\right\|, \quad \psi \in \mathscr{D}\left(\mathrm{H}_{0}\right) .
$$


Therefore, the integral $\mathrm{I}_{0}(\psi, \tau)$ exists and so for the Möller operator

$$
\Omega_{t_{0}}\left(\mathrm{H}, \mathrm{H}_{0}\right)=\underset{t \rightarrow+\infty}{\mathrm{s}-\lim _{t \rightarrow \infty}} U\left(t, t_{0}\right)^{-1} e^{-i\left(t-t_{0}\right) \mathrm{H}_{0}} .
$$

By Theorem 2, we proved the existence of $\Omega_{t_{0}}\left(\mathrm{H}_{\mathrm{A} t}, \mathrm{H}\right)$ and $\Omega_{t_{0}}\left(\mathrm{H}, \mathrm{H}_{\mathrm{A} t}\right)$ so that one might think that by the chain rule theorem [13], one would derive the existence of the Möller operator $\Omega_{t_{0}}\left(\mathrm{H}_{0}, \mathrm{H}\right)$. This is true only if

$$
\Omega_{t_{0}}\left(\mathrm{H}_{0}, \mathrm{H}_{\mathrm{A} t}\right)=\underset{t \rightarrow+\infty}{\mathrm{s}-\lim _{t \rightarrow+}} e^{i\left(t-t_{0}\right) \mathrm{H}} e^{-i\left(t-t_{0}\right) \mathrm{H}_{\mathrm{A} t}}
$$

exists in which case

$$
\Omega_{t_{0}}\left(\mathrm{H}_{0}, \mathrm{H}\right)=\Omega_{t_{0}}\left(\mathrm{H}_{0}, \mathrm{H}_{\mathrm{A} t}\right) \Omega_{t_{0}}\left(\mathrm{H}_{\mathrm{A} t}, \mathrm{H}\right) .
$$

However, in general, it is only the generalized wave operator

$$
\Omega^{\prime}\left(\mathrm{H}_{0}, \mathrm{H}_{\mathrm{A} t}\right)=\underset{t \rightarrow+\infty}{s-\lim _{t \rightarrow}} e^{i t \mathrm{H}_{0}} e^{-i t \mathrm{H}_{\mathrm{A} t}} \mathrm{P}_{\mathrm{C}}
$$

which exists and then one would have the existence of $\Omega\left(\mathrm{H}_{0}, \mathrm{H}\right)$ only if the range of $\Omega_{t_{0}}\left(\mathrm{H}_{\mathrm{A} t}, \mathrm{H}\right)$ is contained in the domain of $\mathrm{P}_{\mathrm{C}}$. But since $\Omega_{t_{0}}\left(\mathrm{H}, \mathrm{H}_{\mathrm{A} t}\right)$ exists on all of $\mathscr{L}^{2}\left(\mathbb{R}^{3}\right)$ the range of $\Omega_{t_{0}}\left(\mathrm{H}_{\mathrm{A} t}, \mathrm{H}\right)$ is $\mathscr{L}^{2}\left(\mathbb{R}^{3}\right)$ so that the chain rule theorem applies only if $\mathrm{P}_{\mathrm{C}}=\mathbb{1}$, that is, if $\mathrm{H}_{\mathrm{A} t}$ has no bound states!

\section{References}

1. Agostini,P., Barjolt, G., Bonnal, J.F., Mainfray, G., Manus, C., Morellec, J.: I.E.E.E. J. Quantum Electron Q.E. 4, 667 (1968)

Agostini,P., Barjolt, G., Mainfray,G., Manus, C., Thebault, J.: I.E.E.E. J. Quantum Electron Q.E. 6, 782 (1970)

Held,B., Mainfray,G., Manus,C., Morellec,C.: Xème conférence sur phenomeca in ionised gases. London (1972), Donald Pearsons, England. Phys. Rev. Lett. 28, 130 (1972)

Van Lu, M., Mainfray, G., Manus, C., Tugov, I.: Phys. Rev. A7, 91 (1973)

Delone, G. A., Delone, N.B., Piskova, G. K.: Zh. Eksp. Teor. Fiz. 62, 1272 (1972); Engl. Trans. Soviet. Physics J.E.T.P. 35, 672 (1972); see also: Xème conférence sur phenomeca in ionised gases. London (1971)

2. Keldysh, L.V.: Zh. Eksp. Teor. Fiz. 47, 1945 (1964) (see also Phys. J.T.E.P. 20, 1307 (1965)

Bebb, H. B., Gold, A.: Phys. Rev. 143, 1 (1968)

Morton, V.M.: Proc. Phys. Soc. 92, 301 (1967)

Gontier, Y., Trahin, M.: Phys. Rev. 4A, 1896 (1971)

3. Reiss, H. R.: Phys. Rev. A 1, 803 (1970); Phys. Rev. D4, 3533 (1971)

Faisal, F.H. M.: J. Phys. B6, L 89 (1973)

4. Yosida, K.: Functional analysis, Chapter XIV, Section 4. Berlin-Heidelberg-New York: Springer 1968

5. Dollard,J.D.: J. Math. Phys. 5, 729 (1964)

6. Simon, B.: Quantum mechanics for Hamiltonians defined as quadratic forms, Chapter II, p. 32. Princeton: University Press 1971

7. Simon, B.: Ibid. Appendix, p. 206

8. Simon, B.: Ibid. Chapter II, p. 54

9. Simon, B.: Ibid. Chapter IV, p. 102

10. Prugovecki,E., Tip, A. : Scattering theory in a time-dependent external field. To appear: J. Phys. A (1974)

11. Dollard, J.D.: J. Math. Phys. 7, 802 (1966) 
12. Buslaev, V.S., Matveev, V.B.: Theor. Math. Phys. 2, 226 (1970)

13. Kato, T.: Perturbation theory for linear operators, Chapter X, $\S 3$, p. 532. Berlin-HeidelbergNew York: Springer 1966

14. Combe, $\mathrm{Ph}$.: Thèse (1974)

Communicated by W. Hunziker

P. Combe

E. Mourre

J.-L. Richard

C.N.R.S.

Physique Théorique

31, chemin J. Aiguier

F-13274 Marseille Cedex 2, France 